\title{
The Study on the Motivation of Sex-Selective Abortion among Indian Immigrants in U.S.A
}

\author{
Jill Tucker ${ }^{1}$, Sung Seek Moon ${ }^{2}$, Minkyung Kim ${ }^{3}$, KyungSook Kim ${ }^{4 *}$ \\ ${ }^{1}$ School of Social Work, University of Arlington, M.S.W. U.S.A \\ ${ }^{2}$ School of Social Work, University of South Carolina, U.S.A \\ ${ }^{3}$ Department of Childwelfare, Namseoul University, Korea \\ ${ }^{4}$ Department of Nursing, Namseoul University, Korea \\ sungmoon@uta.edu,mkm35@nsu.ac.kr,Kgs4321@nsu.ac.kr
}

\begin{abstract}
This qualitative study sought to explore the motivations behind sex-selective abortion among Indian immigrants in the United States. Using a theoretical framework incorporating social learning theory and self-determination theory, the research design consisted of semi-structured interviews of 20 married Indian immigrant women. Based on an analysis of these interviews, the following emergent themes were discovered: The extrinsic motivation was "Money", "Education (lack of)", "Familial Pressure", and the introjected motivation was "Problems for and from Girls", "Dowry", "Familial Preference", and identified motivation was "Familial Ties", "Inheritance", "Son's Duty", "Name Carried Forward", and the intrinsic motivation was "Want a Boy". Theoretical and social work practice implications based on the findings of the study were discussed. Based on the researcher's findings, there remains a great deal of opportunity for future studies to provide more insight into this phenomenon. Beyond researching this specific issue, the researcher recommends a continued focus on women's rights issues within the United States.
\end{abstract}

Key Words: Selective abortion, Indian immigrants, Gender, Son preference

\section{Introduction}

In the 1970s and 80s, the cultural preference for sons coupled with the introduction of modern methods of prenatal sex determination led to sex-selective abortions becoming "the primary method used to alter the sex composition of children" [1].

Many studies have examined the possible motivations behind son-preference in India. While each focuses on different aspects of Indian culture, virtually every study notes the extremely patriarchal system within which India operates. Almond and Edlund's analysis of the 2000 U.S. Census information examined sex ratio data in relation to birth order [2]. Their study found the sex ratio between firstborn children of Asian immigrants to be consistent with that of the general population. However, in second-born children the ratio rose to 1.17 boys born for every one girl, if the first child was a girl. In families with three children the ratio climbed to 1.51 boys for every one girl, if the first two children were female [2].

Additionally, Abrevaya's study used both U.S. Census and federal natality data to examine this issue among specific ethnic groups [3]. The study showed findings similar to Almond and Edlund's study (2008), specifically noting higher percentages of male births

\footnotetext{
* Corresponding Author

Department of Nursing Science, Namseoul University,

91 Daehak-ro Seonghwan-eup Sebuk-gu Cheonan-si Chungcheongnam-do S. KOREA.

E-mail: kgs4321@nsu.ac.kr
} 
among Chinese, Indian, and Korean populations, with the most marked increase among Indian and Chinese families [3].

One might assume that Indians who immigrate to the United States would be immune to the issues that have been shown to increase sex-selective abortion in India. However, urban areas in India have actually demonstrated higher sex ratios than rural villages. Additionally, statistics have shown a "positive linkage between abnormal sex ratio and better socio-economic status and literacy" [1]. This suggests that increases in standards of living and urbanization are not sufficient to change the role women play in modern society.

Additionally, multiple studies have cited the advent of modern medical procedures such as amniocentesis and ultrasound as a contributing factor to the increase in female feticide [1]. Zhou and his colleagues [4] deemed such technologies to be "the single most important factor responsible for decrease in sex ratios and increase in female feticides". The United States has access to some of the premier medical procedures in this area. It is therefore unlikely that this problem of disproportionate female births among certain immigrant populations will disappear on its own. Rather, modern scientific advancements could compound the problem.

Additionally, cultures with higher preference for sons value hyper-masculinity and reinforce stereotypes about male and female roles [5]. This could lead to unhealthy male/female relationships in the future, and problems of domestic violence. With both parties feeling that men have the right to abuse women simply because of their sex, and women being traditionally blamed for being unable to give their husbands a male heir [67], women in cultures who value sons over daughters potentially face problems across their lifetime. If the issue of sex-selective abortion transcends immigration to the United States, it is likely that other manifestations of gender discrimination could persist.

\section{Theoretical Framework}

The current research operated on a dualistic theoretical framework. By combining social learning theory and self-determination theory, this study explored both the transmission of and motivation behind sex-selective abortion.

Social learning theory emphasizes two distinct methods of learning: learning through response consequences and learning through modeling [8]. Simply stated, this theory states "most social learning is accomplished through observation and modeling behavior" [8]. Social learning theory asserts that an individual who models a behavior makes a conscious decision to do so. This self-motivation component to changes in behavior is a key difference between social learning theory and other theories of behaviorism [8]. It is important to note, however, that social learning theory only shows externalizations of ideologies, and not internalizations. An individual does not have to agree with the ideology to exhibit its physical manifestations. To examine the internalization of a given ideology, one turns to self-determination theory, which says that understanding motivation is essential in determining whether a belief has been internalized or not. Selfdetermination theory divides motivations into categories along a continuum ranging from extrinsic to introjected to identified to intrinsic motivation [9-10].

Social learning theory would say that Indian immigrants in the United States, who practice sex-selective abortion, do so because they are modeling a behavior exhibited either in popular media or by friends and family members in India. While social learning theory can explain how the transmission of this behavior occurs, it does not explain whether Indian immigrants retain the actual preference for sons, which originally motivated the practice in India. Self-determination theory allows for an explanation of why someone would engage in sex-selective abortion and/or son preference. Based on the review of literature related to the postulates of social learning theory and selfdetermination theory, the research questions for this study are as follows: 
1. What type of motivation do Indian immigrants give for engaging in sex-selective abortion?

2. What type of motivation do Indian immigrants perceive for others engaging in sexselective abortion?

\section{Materials and Methods}

\subsection{Sampling and Data Collection}

A participant was considered a first-generation immigrant if she had lived in India and immigrated to the United States either as a child or as an adult. A total of 24 women agreed to participate in this research. Four of the participants, who initially agreed to be interviewed did not meet the study's criteria and were ineligible to participate. The final sample size was 20 participants. The sample was recruited through convenience and snowball sampling via the researcher's personal contacts within the Indian immigrant community. Those contacts were asked to refer women, who fell within the age limit and marital status requirements of the study. An application to the Institutional Review Board (IRB) was made by the first author. The interview schedule as well as the research protocol were reviewed and approved by the IRB. Participants, who agreed to be interviewed, were asked to sign the consent form, which was promptly placed in a separate folder from their response sheet. Interviews were conducted in private locations chosen by the participants.

\subsection{Measures and Data Analysis}

The interview contained thirteen demographic questions. These questions were intended to gather information on a possible relationship between socio-economic status, acculturation, religion, and education-level on sex-selective abortion. Additionally, participants were asked seven questions related to their direct and indirect exposure to son preference and sex-selective abortion. Finally, participants were asked if they have ever engaged in sex-selective abortion. If participants answered in the affirmative, they were asked, When you had a sex-selective abortion, what was your reason? If participants denied having engaged in sex-selective abortion, they were asked if they have ever considered doing so. All participants were asked to speculate as to why someone might choose to have a sex-selective abortion.

The researcher used ATLAS.ti, a qualitative data analysis software, to code the participants' responses and look for emerging themes. Once all interviews had been completed and transcribed, the researcher began data analysis. The researcher spent a substantial amount of time reading each transcription and becoming familiar with the data. Throughout the process of becoming familiar with the data, the researcher made note of repeating ideas. After the initial coding phase was completed, the researcher looked at each emerging theme separately and read the coded segments of each theme to check for consistency within the themes. After developing a finalized list of emerging themes from the interviews, the researcher analyzed the data and determined where each theme should be placed on the motivational continuum described by self-determination theory.

\section{Results}

\subsection{Characteristics of Participants}

A total of 20 women participated in this study and participants' age ranged from 23 to 44 years (Mean $=31.75$ years), with eight $(40 \%)$ participants between the ages of 23 and 29 , nine $(45 \%)$ between the ages of 30 and 39, and three(15\%) between the ages of 40 and 44 years old. Participants' length of marriage ranged from five months to 25 years 
(Mean $=7.89$ years). Three participants $(15 \%)$ had been married for less than a year; seven (35\%) had been married between one and five years; four $(20 \%)$ had been married between six to ten years; two(10\%) had been married between 11 and 15 years; two(10\%) had been married for 16 to 20 years; and two(10\%) had been married for 21 to 25 years. Ten participants (50\%) had no children; eight participants (40\%) had two children; one participant $(5 \%)$ had three children; and one participant $(5 \%)$ had four children. All participants with children $(\mathrm{n}=10)$ had at least one daughter. Amongst the participants with children $(n=10)$, only one participant did not have any sons; one participant had multiple sons; and three participants had multiple daughters.

\subsection{Speculation about Motivation}

Participants were asked to speculate the reason someone might give for engaging in sex-selective abortion. This open-ended question yielded the most in-depth responses from participants. With the assistance of ATLAS.ti, the researcher used an open-coding method to search for emerging themes within participants' responses. Based on this opencoding method, the following repeating ideas emerged: "Problems for and from Girls", "Dowry", "Education (lack of)", "Familial Pressure", "Familial Preference", "Familial Ties", "Inheritance", "Son's Duty", "Money", "Name Carried Forward", and "Want a Boy".

The theme of "Money" included any mention of general poverty or the cost associated with having children (outside of dowry, which is given its own theme). Financial concerns were cited by eight participants as a reason why someone might choose to have a sexselective abortion.

Education (lack of), this theme pertains to any mention of a lack of education as well as a general lack of knowledge or awareness as a reason someone might engage in a sexselective abortion. Ten participants gave this possible motivation. Many spoke of "backward" or "narrow-minded" people as being the ones, who engaged in sex-selective abortion. In the mind of these participants, sex selective abortion was motivated by a blind obedience to a prescribed standard of rules and regulations in society: "Their thinking is not as open as we are. They still bear narrow-minded thinking and they just follow a set of rules, where the man says, "Give dowry", so I will just follow him.

The theme of familial pressure deals with the sense of obligation a woman might feel towards her husband, parents or in-laws to have a male child. While the theme of "Familial Preference" holds similar ideas, the participant quotations chosen for this theme conveyed a sense of absolute necessity and lack of choice on the part of the woman. According to the seven participants, who cited this possible motivation, a woman would not be acting out of avoidance of shame from her family, but rather because it was absolutely expected of her. One participant described this pressure by stating, "She has no powers, no control to decide what she wants to do. She does not know whether it is[abortion] a good or a bad thing" (P7).

Familial Preference deals with the obligation a woman might feel towards her family to have a son. However, this theme differs from that of "familial pressure" in that the obligation appears to be coming out of an avoidance of shame or a seeking of family approval rather than coercion. Three participants spoke of this specific family-related issue. For example, P1 spoke of an exchange between her aunt and her grandmother after her aunt gave birth to a second girl child, stating, "She [the grandmother] actually mentioned that maybe they should give away the baby. She actually said that within, I think, a few minutes of my aunt giving birth. Obviously, my aunt and my uncle were very upset. And they did not, obviously, pay attention to that" (P1).

Dowry referred to any mention of expense associated with marriage as well as the use of the actual word "dowry". Nine participants cited dowry as a reason for son preference in Indian society: "Usually in India, in my country, they usually have female infanticide because it takes a lot of money to get a female married in your family" (P8). One might 
assume that dowry was an irrelevant issue for Indian immigrants. However, as one participant noted, dowry can and does follow immigrants when they leave their native land.

Problems for and from Girls: Eight participants spoke specifically of the unique problems faced by girls in society and cited that as a possible reason, because of which someone might not want a female child. Specifically, participants spoke about concerns over domestic violence, and a general lack of control over one's future after marriage. One participant stated, "None of the parents would like their daughter to be abused in any way. Nice husband, nice family- What if she does not find?" (P16). Another noted the difference in control, which parents have on female children as compared to male children: "But for girl nothing is in control. If they [the parents] want to see their girl happy, if they want to see their boy happy, they can make the boy happy. However, if they want to see their girl happy, it depends on the in-laws. It depends on girl's in-laws and how they are" (P20).

The theme of "Familial Ties" specifically referred to any mention of daughters moving to another family when they get married or not coming back to their birth family after moving out. Ten participants noted this reasoning. According to participants, after a daughter's marriage, she is almost considered estranged from her biological family. The idea of daughters being completely cut off from their biological family after marriage came through in many participants' choice of language. One participant noted, "Because in India the daughters are given away. She goes to another family and she is no longer a part of the family" (P9).

Inheritance: Only three participants mentioned inheriting property etc. from the family as a reason to have sons. The participants cited inheritance as a motivation for sexselective abortion and couched it in terms of making sure one's wealth stayed within the family

Son's Duty: According to participants, sons are expected to contribute financially to their parents' well-being. Additionally, they are expected to take care of their parents in their old age. Thirteen participants included this idea in their reasons, which someone might choose to have a sex-selective abortion, by saying things such as: "And they like male children because they represent the family. They earn money and come back home. That is really a silly reason, I think, but that is the reason" (P8). Additionally, a few participants noted that a married son brings the added bonus of having a wife to help him perform his duties as a son. As these two participants stated, daughter-in-laws contribute to their in-laws' well-being. However, the credit still goes to the son.

Name Carried Forward:Corresponding to the idea of son's being the keepers of the family, which becomes the specific idea of males carrying the family name. Eight participants attested to the importance of preserving a specific lineage, making statements such as, "I think specifically in Indian culture, and in a lot of other cultures, the son carries the family name, and I think that isa big deal" (P2).

In addition to speaking about a son's ability to carry the family name, a few participants talked specifically about a daughter's inability to carry her family name forward: "The boy carry the name for the generation to generation. Whereas, the girl is just, go away, you know? Take away the name from the father" (P15). One participant speculated as to why the issue of lineage might persist even in the Indian immigrant population

Want a Boy: This theme reflects instances when the motivation for son preference and/or sex-selective abortion was cited as simply wanting a boy or not wanting a girl. Only two participants made statements that fit into this category, stating, "My husband has a friend who works with him and it has been a year they had a baby boy. He really wanted a baby boy. He told his wife that he does not want a baby girl. He wants a baby boy" (P1), and, "They had one girl before that and they did not want a second girl and they aborted" (P9). 


\section{Discussion}

Extrinsic Motivation is motivation involving "rule following" and "avoidance of punishment" [11]. The themes of "Money", "Education" and "Familial Pressure" were considered to be fitting into this category of motivation.

Participants frequently spoke about financial constraints as an issue affecting one's family size and willingness to have daughters. Their explanations of why someone might engage in sex-selective abortion imply that if people were not impoverished, they would afford to have a larger family. However, it is important to note that while money would allow people to have more children and might curb the practice of sex-selective abortion, it would not do anything to change the issue of son preference.

Education was placed under extrinsic motivation because, like with the theme of money, participants considered this to be a circumstantial contributor to the issue of sexselective abortion. Participants' explanations suggested that a lack of exposure to ideas of gender equality as well as a lack of understanding of the dangers associated with abortion were perpetuating the practice. The theme of Familial Pressure highlighted the coercion, which many women face by spouses or in-laws to have a sex-selective abortion. Familial Pressure fits into this category of motivation because of the absolute expectation that these women obey their family members' wishes regarding their pregnancy.

Ryan and Connell [11] define introjected motivation as "internal, esteem-based pressures to act, such as avoidance of guilt and shame or concerns about self- and otherapproval". The researcher identified the themes of "Problems for and from Girls", "Dowry" and "Familial Preference" as representing introjected motivation.

"Problems for and from Girls" demonstrates an introjected motivation because of the participants' frequent mention of feeling either guilt towards their daughters for not being able to provide them with a good life or fear of shame as a result of their daughters' premature loss of virginity. The theme of "Dowry" also represents an introjected motivation due to the strong attachment that dowry has to a family's standing in society. While technically an illegal practice in India, dowry is still expected as a way to ensure that one's daughter marries into a desirable family."Familial Preference" is similar to the theme of "Familial Pressure". However, the difference between the two themes is the level of coercion involved.

Identified motivation occurs when one's actions are "valued and judged as important for an individual, and especially that it is perceived as chosen by oneself" [12]. The researcher placed the themes of "Familial Ties" "Inheritance" "Son's Duty" and "Name Carried Forward" in this motivational category.

The theme "Familial Ties" was considered to fall under "identified motivation" because participants who talked about this concept spoke in terms of sons being more connected to their parents as compared to daughters. Family connectedness reflects an important value in Indian society, so a daughter's inability to connect makes her less valuable in many people's eyes.

"Inheritance" represents an identified motivation because it reflects the desire to keep one's wealth within a family line. Participants who cited inheritance as a reason for son preference spoke about ensuring that one's own possessions do not go to another household. While parents do not receive any personal benefit from keeping their wealth within their own family, the pride that comes with establishing a prosperous estate certainly provides a personal benefit to them.

"Son's Duty" clearly falls under the category of identified motivation. Desiring a son because of the cultural expectation that a son will contribute to his family through financial assistance and physical care represents a strong personal interest on the part of parents.

Likewise, the theme of "Name Carried Forward" demonstrates a parent's desire to see their lineage continue. Just as the theme of "Inheritance" illustrates a parent's desire to 
produce some type of legacy for the future, the theme of "Name Carried Forward" shows the importance placed on ensuring that one's posterity maintains a certain standing in society.

The category of "intrinsic motivation" was the only category without a substantial number of responses fitting into it. Intrinsic motivation typically includes behaviors "done simply for [their] inherent enjoyment or for fun" [11].

The researcher chose to keep the intrinsic motivation category since it is possible for someone to say that they derive pleasure from having male children for no reason other than that they prefer sons. The theme of "Want a Boy" was the only theme, which the researcher felt truly embodied this type of motivation. Only two participants gave responses that fitted into this theme. However, this could be due to participants' difficulty with placing themselves in the position of a person engaging in a sex-selective abortion. The strong moral judgment and statements of disbelief that anyone could abort a fetus based upon its sex could prevent participants from providing a genuine preference for sons as the reason why someone might have a sex-selective abortion.

\section{Acknowledgments}

This research was supported financially by Namseoul University in 2015.

\section{References}

[1] C. Guilmoto, "Characteristics of Sex-Ratio Imbalances in India and Future Scenarios", Paper presented at the United Nations Population Fund's $4^{\text {th }}$ Asia Pacific Conference on Sexual and Reproductive Health, Hyderabad, India, (2009).

[2] D. Almond and L. Edlund, "Son biased sex ratios in the 2000 United States Census", In Lee, R. (Ed.). Proceedings of the National Academy of Sciences of the United States of America (PNAS), (2008).

[3] J. Abrevaya, "Are there missing girls in the United States? Evidence from birth data. Manuscript in preparation", University of Texas at Austin, (2009).

[4] C. C. Zhou, X. L. Wang, W. J. Zheng, X. D. Zhou, L. L. Li and T. T. Hesketh, "The high sex ratio in China: What do the Chinese think?", Journal of Biosocial Science, vol. 44, no. 1, (2012), pp. 121-125.

[5] R. Mahalingam and S. Balan, "Culture, son preference, and beliefs about masculinity", Journal of Research on Adolescence, vol. 18, no. 3, (2008), pp. 541-553.

[6] S. Mitra, "Domestic violence along with its sociocultural determinants among pregnant women attending $\mathrm{MCH}$ clinic of a subdivisional hospital in West Bengal", Indian Journal of Community Medicine, vol. 31, no. 4, (2009), pp. 267-269.

[7] J. E. Yi, "Unwanted daughters: Psychological implications of son preference on Korean women, Dissertation Abstracts International", vol. 73, no. 11, (2011).

[8] D. L. Flor, "A comparative approach to the internalization of religiousness in preadolescent youth (Doctoral dissertation, University of Georgia, 1998)", Dissertation Abstracts International, vol. 59, no. 4, (1998).

[9] E. L. Deci, H. Eghrarl, B. C. Patrick and D. R. Leone, "Facilitating internalization: The selfdetermination theory perspective", Journal of Personality, vol. 62, no. 1, (1994), pp. 120-142.

[10] G. Roth, A. Assor, Y. Kanat-Maymon and H. Kaplan, "Assessing the experience of autonomy in new cultures and contexts", Motivation and Emotion, vol. 30, (2006), pp. 365-376.

[11] R. M. Ryan and J. P. Connell, "Perceived locus of causality and internalization: Examining reasons for acting in two domains", Journal of Personality and Social Psychology, vol. 57, (1989), pp. 749-761.

[12] R. J. Vallerand, L. G. Pelletier, M. R. Blais, N. M. Briere, C. Senecal and E. F. Vallieres, "The Academic Motivation Scale: A measure of intrinsic, extrinsic, and amotivation in education", Educational and Psychological Measurement, vol. 52, (1992), pp. 1003-1017. 
International Journal of Bio-Science and Bio-Technology

Vol.7, No.3 (2015) 